\title{
Adding a nurse-based intervention programme to usual care improves manic symptoms in people with bipolar disorder
}

Simon GE, Ludman EJ, Unutzer J, et al. Randomized trial of a population-based care program for people with bipolar disorder. Psychological Med 2005;35:13-24.

Does adding a nurse-based intervention programme to usual care improve the effectiveness of treatment for bipolar disorder?

\section{METHODS}

Design: Randomised controlled trial.
Follow up period: Twelve months.
Selting: Four health clinics in the USA; August 1999 to October
Patients: 441 people treated for bipolar disorder type I or II
(DSM-IV) in the previous 12 months. People were excluded if
cognitive impairment prevented giving informed consent.
Intervention: Usual care, or usual care plus a nurse-based
intervention programme. The intervention programme consisted
of an initial patient assessment and treatment planning session,
followed by monthly telephone monitoring of mood symptoms
and medication with the support of the treating mental healthcare
team, together with a group psycho-educational programme.
Outcomes: Psychiatric status ratings for mania and depression.

\section{MAIN RESULTS}

Usual care with a nursed-based intervention programme reduced manic, but not depressive symptoms, compared with usual care alone, over 12 months (mania scores: $\mathrm{p}=0.025$; depressive scores: $\mathrm{p}=0.82$; actual values not stated).

For correspondence: Dr Gregory Simon, Center for Health Studies, 1730 Minor Avenue, \#1600 Seattle, WA 98101, USA; simon.g@ghc.org

Sources of funding: Grant R01 MH59125 from the National Institute of Mental Health.

\section{CONCLUSIONS}

Usual care with a nurse-based intervention programme is more effective than usual care alone for treating bipolar disorder.

\section{Commentary}

7 reatments demonstrated to be efficacious for bipolar disorder in clinical trials deliver disappointing results when applied in general practice. Rather than searching for a more efficacious molecule, Simon et al conducted a well powered randomised controlled trial comparing outcomes over a 12 month period for bipolar patients continuing usual care or adding a multicomponent systematic care management programme coordinated by a specialised nurse.

The results indicate that tightening up the intervention-measurementmanagement feedback loop is beneficial. The programme consistently produced a statistically significant reduction in average mania severity scores and a modest reduction in depression over time. Both groups, however, enjoyed rather striking reductions in symptom severity. As the authors point out, this is unlikely to represent regression to the mean, because the sample was not selected for high symptom acuity at study entry. Thus participation in study procedures probably had a salutary effect on the course of bipolar illness. It is plausible that serial independent outcome assessments represent an active interventionenhancing effectiveness. Benefit might be derived from other study procedures or leakage of improved approaches to usual care.

The results are consistent with non-specific effects observed in clinical trials $^{12}$ and encourage us to integrate more coordination and measurement into our management of bipolar disorder while we wait for research to clarify the active components. Although a nurse specialist implementing the full systematic care programme might best serve this function, the identification of a care partner, appointment reminders, and quarterly independent assessment might represent a feasible set of core interventions for nearly all mental health settings.

Gary S Sachs, MD Associate Professor of Psychiatry, Harvard Medical School and Director, Bipolar Clinic and Research Program, Massachusetts General Hospital, Boston, MA, USA

1 Bowden CL, Calabrese JR, McElroy SL, et al. A randomized, placebocontrolled 12-month trial of divalproex and lithium in treatment of outpatients with bipolar I disorder. Divalproex Maintenance Study Group. Arch Gen Psychiatry 2000;57:481-9.

2 Rucci P, Frank E, Kostelnik B, et al. Suicide attempts in patients with bipolar I disorder during acute and maintenance phases of intensive treatment with pharmacotherapy and adjunctive psychotherapy. Am J Psychiatry 2002;159:1160-4. 